\title{
FIBRE BUNDLES OVER SPHERES
}

\author{
RYOJI SHIZUMA
}

This note is concerned with homotopy relations in fibre bundles over spheres, in connection with my previous note. ${ }^{1)}$

1. Let $R$ be a fibre boundle, ${ }^{2)}$ with the $n$-dimensional sphere $S^{n}$ as the base space and with fibres which are connected and simple in $n-1$ dimension. ${ }^{3)}$ Let further $\pi$ denote the projection of $R$ onto $S^{n}$. We take a simplicial decomposition $K$ of $S^{n}$ which is so fine that the star of each simplex lies in a coordinate neighborhood of $S^{n}$. It is possible to define a continuous map $\psi$ of $S^{n}$ in $R$ such that $\pi \psi(p)=p$ for $p \in S^{n}$, with exactly one singular point 0 . We may regard 0 as an inner point of an $n$-simplex $T$ of $K$. We decompose $\pi^{-1}(T)$ into the topological product of $T$ and a fixed fibre $F$, and denote by $\lambda$ the projection of $\pi^{-3}(T)$ onto $F$. Since the map $\lambda \psi$ is defined over the boundary $\dot{T}$, we get a map of a sphere $S^{n-1}$ into $F$ and hence an element $\alpha$ of the homotopy group $\pi^{n-1}(F)$ of $F$. The element $\alpha$ is a topological invariant of $R$, which I was referred to as the characteristic number. ${ }^{4)}$ In particular, if $R$ is the fibre bundle of non-zero tangent vectors of $S^{n}$, the characteristic number is nothing else than the EulerPoincaré characteristic of $S^{n}$.

We now proceed to prove the following

Theorem 1. Let $R$ be a fibre bundle over $S^{n}$, and $F$ the fibre. Let further $\alpha$ be the characteristic number of $R$. If $F$ is simple in $n-1$ dimension, we have

$$
\pi^{n-1}(R)=\left(\pi^{n-1}(F)\right)_{\alpha},
$$

where the right hand side stands for the factor group of $\pi^{n-1}(F)$ by the subgroup generated by $\alpha$.

Proof. We first consider the homotopy sequence

$$
\pi^{n}(R, F) \rightarrow \pi^{n-1}(F) \rightarrow \pi^{n-1}(R) \rightarrow \pi^{n-1}(R, F) .
$$

Since $\pi^{n-1}(R, F)=\pi^{n-1}\left(S^{n}\right)=0, \pi^{n-1}(F) \rightarrow \pi^{n-1}(R)$ is onto homomorphism. A

Received February 20, 1950.

1) R. Shizuma [4].

2) We shall adopt the definition of Steenrod and follow his terminology. See N. Steenrod [5].

3) S. Eilenberg [1].

4) R. Shizuma [4]. 
map $f$ of an $n$-cell $E^{n}$ in $R$ such that $f\left(\dot{E}^{n}\right) \subset F_{0}$, where $F_{0}$ is the fibre over the point 0 , defines an element of $\pi^{n}(R, F)$, denoted by [f]. The map $\psi$ indicated above defines an $n$-cell $V$ in $R$, while $\dot{V}$ determines an element $\alpha$ of $\pi^{n-1}(F)$. Clearly, $f$ determines a degree $m$. Since the isomorphism between $\pi^{n}(R, F)$ and $\pi^{n}\left(S^{n}\right)$ is given by the correspondence $[f] \rightarrow m^{5}$, we find

$$
[f]-m[\psi]=0 \text {. }
$$

Thus the homomorphism $r: \pi^{n}(R, F) \rightarrow \pi^{n-1}(F)$ given in turn

$$
r[f]-\operatorname{mr}[\psi]=0 .
$$

Since $r[\psi]=\alpha$, we have $r[f]=m \alpha$. Hence we have

$$
\pi^{n-1}(R)=\left(\pi^{n-1}(F)\right)_{\alpha} .
$$

2. As an application we shall give a simpler proof of a result obtained by Stiefel. Let $V(n, r)$ be the manifold of $r(1 \leqq r \leqq n)$ unit orthogonal vectors through the origin of the Euclidean $n$-space $E^{n}$. Let $R^{n}$ be the group of all rotations of $S^{n} . \quad V(n, r)$ is taken to be a homogeneous space $R^{n-1} / R^{n-r-1}$. From now on we agree that all the homology groups are concerned with respect to integer cofficients.

THEOREM 2 (Stiefel).6) All the homology groups of dimension $<n-r$ of $V(n, r)$ vanish and the homology group $B^{n-r}(V(n, r))$ of dimension $n-r$ is the free cyclie group or the cyclic group of order 2 according as the following condition is satisfied or not: $n-r$ is even or $r=1$.

Proof. Since $V(n, 1)=S^{n-1}$, it follows that $B^{n-1}(V(n, 1))$ is free cyclic and $B^{s}(V(n, 1))=0$ for $s<n-1$.

Suppose now $r \neq 1$. We observe that $V(n, r)=R^{n-1} / R^{n-r-1}$ may be regarded as a fibre bundle over $S^{n-1}$ with fibres homeomorphic to $V(n-r, r-1)$. Since $n^{s}\left(S^{n-1}\right)=0$ for $s<n-1$, any map of $S^{s}$ in $S^{n-1}$ is contractible into a point. If $S^{s}$ is mapped in $S^{n-1}$ through $V(n, r)$, the covering homotopy deforms $S^{s}$ into $V(n-1, r-1)$. If the image of $S^{s}$ in $V(n-1, r-1)$ is contractible in $V(n, r)$, it is contractible in $V(n-1, r-1)$, providing $s<n-2$. Hence $\pi^{s}(V(n, r))$ $=\pi^{s}(V(n-1, r-1))$ for $s<n-2$. Repeating the same argument, we have finally $\pi^{s}(V(n, r))=\pi^{s}(V(n-r+2,2))$ for $s \leqq n-r$. Moreover, it follows that, if $s<n-r$,

$$
\pi^{s}(V(n, r))=\pi^{s}(V(n-r+1,1))=\pi^{s}\left(S^{n-r+1}\right)=0 .
$$

Hence we have

5) See W. Hurewicz and N. Steenrod [3], Theorem 2.

6) See E. Stiefel [6]. 


$$
\pi^{s}(V(n, r))=0 \text { for } s<n-r
$$

and

$$
\pi^{n-r}(V(n, r))=\pi^{n-r}(V(n-r+2,2)) .
$$

We notice that $V(n-r+2,2)$ is a tangent sphere bundle over $S^{n-r+1}$. It follows that its characteistic number $\alpha$ is equal to the Euler-Poincare characteristic of $S^{n-r+1}$. Consequently $\alpha$ has the value 2, if $n-r$ is odd, and 0 , if $n-r$ is even. Since, by Theorem 1 , we have

$$
\pi^{n-r}(V(n-r+2,2))=\left(\pi^{n-r}\left(S^{n-r}\right)\right)_{\alpha},
$$

$\pi^{n-r}(V(n-r+2,2))$ is the free cyclie group or the cyclic group according as $n-r$ is even or not. On the other hand, it follows from Hurewicz's theorem," that $\pi^{s}(V(n, r))(s \leqq n-r)$ can be considered to be identical with the $s$-th homology group $B^{s}(V(n, r))$ with integer coefficients. Thus the theorem is completely proved.

Let $U(n, r)$ be the manifold of $r(1 \leqq r \leqq n)$ linearly independent vectors in a complex vector space of $n$ complex dimensions. The corresponding result in the complex case can be deduced in an analogous way, using the unimodular unitary groups instead of the rotation groups. In fact, it can be easily verified that all homology groups of dimension $<2 n-2 r+1$ of $U(n, r)$ vanish and that the homology group $B^{2 n-2 r+1}(U(n, r))$ of dimension $2 n-2 r+1$ is a free cyclic group.

\section{BIBLIOGRAPHY}

[1] S. Eilenberg, On the relation between the fundamental group of a space and the higher homotopy groups, Fund. Math., 32 (1939), 167-175.

[2] W. Hurewicz; Beiträge zur Topologie der Deformationen II, Proc. Akad. Amsterdam, 38 (1935), 521-528.

[3] W. Hurewicz and N. Steenrod, Homotopy relations in fibre spaces, Proc. Nat. Acad. Sci. U.S. A., 27 (1941), 60-64.

[4] R. Shizuma, Homotopy properties of fibre bundles, forthcoming J. of Math. of Japan.

[5] N. Steenrod, The classification of sphere bunbles, Ann. of Math., 45 (1944), 294-311.

[6] E. Stiefel, Richtungsfelder und Fernparallelismus in $n$-dimensionalen Mannigfaltigkeiten, Comment. Math. Helv., 8 (1936), 3-51.

\section{Nagoya University}

i) See W. Hurewicz [2], Theorem 1 